\title{
Níveis de Sódio para Poedeiras Comerciais no Primeiro e Segundo Ciclos de Produção Alice Eiko Murakami ${ }^{1}$, Denise Fontana Figueiredo², Adriana Zeponi Peruzzi ${ }^{4}$, José Rodrigo Galli Franco ${ }^{3}$, Márcia Izumi Sakamoto ${ }^{3}$
}

\begin{abstract}
RESUMO - O objetivo deste trabalho foi avaliar os níveis de sódio e balanços eletrolíticos da dieta (BED) sobre desempenho produtivo e a qualidade externa dos ovos de poedeiras comerciais no primeiro e segundo ciclos de produção. Foram realizados dois experimentos com duração de 112 dias cada, divididos em quatro ciclos de 28 dias. No experimento I, utilizaram-se 160 poedeiras Lohmann com 25 semanas de idade e no experimento II, 160 poedeiras Babcok pós-muda forçada, com 80 semanas de idade. O delineamento experimental foi inteiramente casualizado, com cinco tratamentos, quatro repetições e oito aves por unidade experimental. As rações foram formuladas à base de milho e farelo de soja, utilizando-se os valores de composição química e energética dos alimentos, segundo Rostagno et al. (1985), e o balanço eletrolítico da dieta foi calculado segundo Mongin (1980). Os tratamentos consistiram de cinco níveis de sódio provenientes do bicarbonato de sódio e sal comum, sendo que para o experimento I os níveis de sódio empregados foram de 0,$12 ; 0,15 ; 0,18 ; 0,21$ e $0,24 \%$ e os balanços eletrolíticos calculados, de 205, 218, 231, 243 e $246 \mathrm{mEq} / \mathrm{kg}$; para o experimento II, foram de 0,$13 ; 0,15 ; 0,17 ; 0,19$ e $0,21 \%$ e 174, 183, 192, 200 e $209 \mathrm{mEq} / \mathrm{kg}$, respectivamente. Foram avaliados os dados referentes ao desempenho produtivo, à qualidade externa dos ovos e porcentagem de umidade das excretas, a qual foi avaliada somente no primeiro experimento. Os níveis de sódio estudados e os balanços eletrolíticos das dietas não influenciaram as variáveis analisadas nos dois experimentos. Portanto, conclui-se que o nível de $0,12 \%$ de sódio, em dietas com $205 \mathrm{mEq} / \mathrm{kg}$, para poedeiras no primeiro ciclo de produção, e de $0,13 \%$ de sódio, em dietas com $174 \mathrm{mEq} / \mathrm{kg}$, para poedeiras no segundo ciclo de produção, foram suficientes para proporcionarem bom desempenho produtivo e boa qualidade externa dos ovos.
\end{abstract}

Palavras-chave: balanço eletrolítico, muda forçada, poedeiras comerciais, sódio

\section{Sodium Levels for Commercial Laying Hens in the First and Second Production Cycles}

ABSTRACT - The aim of this experiment was to verify the sodium levels and electrolyte balance of diets for commercial laying hens on the first and second production periods. Two experiments with 112 days each, divided in four periods of 28 days, were carried out. One hundred and sixty Lohmann laying hens ( 25 weeks old) and 160 Babckok forced molting laying hens ( 80 weeks old), in the first and second experiments, respectively, were used. The laying hens were assigned to a completely randomized design, with five treatments, four replicates and eight birds each. The diets were formulated based on corn and soybean meal and the values of chemical and energy feeds composition were calculated according to Rostagno et al. (1985) and the electrolyte balance of diet was calculated according to Mongin (1980). Five sodium levels, from common salt and sodium bicarbonate, were used. For the first experiment, the sodium levels were $0.12 ; 0.15 ; 0.18 ; 0.21 ; 0.24 \%$ and the calculated electrolyte balance was $205,218,231,243,246 \mathrm{mEq} / \mathrm{kg}$ and for the second one $0.13 ; 0.15$; $0.17 ; 0.19 ; 0.21 \%$ and $174,183,192,200,209 \mathrm{mEq} / \mathrm{kg}$, respectively. At the end of each experimental period, data of productive performance, eggshell quality and excreta moisture were evaluated, and the last one was evaluated only in the first trial. The evaluated variables, in all experiments, were not influenced by sodium levels and dietary electrolyte balance. Therefore, it can be concluded that sodium level of .12\%, for $205 \mathrm{mEq} / \mathrm{kg}$ diets, for laying hens in the first production period and the sodium level of $0.13 \%$, for $174 \mathrm{mEq} / \mathrm{kg}$ diets, for laying hens in the second production cycle, were sufficient to provide a good productive performance and good egg shell quality.

Key Words: electrolyte balance, forced molting, laying hens, sodium

\section{Introdução}

O ovo caracteriza-se por ser um alimento de elevado valor nutritivo, com proteína de alto valor biológico, que já vem naturalmente embalado e, portanto, a casca tem grande importância na qualidade do ovo sob o ponto de vista de conservação do seu valor nutritivo e comercialização.

Uma das características de qualidade que mais pesam para o produtor de ovos é justamente a consistência da casca. Estima-se que perdas de ovos trincados e/ou rachados estejam compreendidas entre 6,0 e 12,3\% ao ano (Vicenzi, 1996). Além disso, à medida que o lote de galinhas envelhece, há queda na

\footnotetext{
1 Professora Dra do Departamento de Zootecnia da UEM. Av. Colombo, 5790 - CEP: 87020-900-Maringá-PR. E.mail: aemurakami@uem.br

2 Doutoranda da FCAVJ - UNESP - Jaboticabal - SP. E.mail: deniseffigueiredo@yahoo.com.br

3 Mestrando (a) do PPZ da Universidade Estadual de Maringá. E.mail: zegalli@yahoo.com.br; mizumiss@hotmail.com

${ }^{4}$ Zootecnista. E.mail: aperuzzi9@hotmail.com
} 
produção e na qualidade interna e externa dos ovos, sendo a muda forçada um manejo economicamente viável para reverter este quadro e aumentar a vida útil da ave.

Para as aves pós-muda, geralmente são utilizadas as exigências do final do primeiro ciclo de postura com uma pequena redução nos níveis dos nutrientes, o que não é nutricionalmente adequado, pois, para um ótimo desempenho dessas aves, torna-se necessária uma revisão quanto às suas exigências nutricionais neste período (Christmas \& Harms, 1983), uma vez que as galinhas poedeiras pós-muda forçada produzem mais de $85 \%$ de ovos tipo grande e extra, contra $70 \%$ das de primeiro ciclo (Oliveira, 1992).

A determinação dos níveis ideais de sódio, cloro e potássio, para cada ciclo de produção da ave é de grande importância, pois estes minerais estão envolvidos em diversos processos fisiológicos, como na manutenção da pressão osmótica, do equilíbrio eletrolítico e balanço ácido-base, dentro dos valores normais. A alta concentração de ânions na dieta (baixo $\mathrm{mEq}$ ) diminui a qualidade da casca dos ovos e baixa o $\mathrm{pH}$, enquanto alta concentração de cátions (alto $\mathrm{mEq}$ ) está associada à melhora da qualidade da casca dos ovos e ao alto $\mathrm{pH}$ no sangue (Miles \& Rossi, 1984).

Apesar da importância de se balancearem adequadamente os eletrólitos da dieta, os mesmos têm recebido pouca atenção dos nutricionistas. Há várias explicações para esta falta de interesse, como o fato de os níveis de potássio estarem quase sempre em excesso nas rações e o sódio e cloro estarem facilmente disponíveis no cloreto de sódio $(\mathrm{NaCl})$ e serem de baixo custo. Contudo, o nível utilizado de $\mathrm{NaCl}$ (sal comum) na ração não é ajustado para estes elementos de acordo com os ingredientes utilizados.

Os efeitos adversos do excesso de cloreto, acima dos níveis necessários, sobre a qualidade da casca do ovo foram relatados por vários pesquisadores e parecem estar relacionados ao efeito da acidificação do cloreto sobre o fluido uterino e sua ação inibitória sobre a anidrase carbônica (Yoselewitz \& Balanave, 1989). No entanto, muitas pesquisas têm procurado minimizar esses efeitos adversos com a substituição do $\mathrm{NaCl}$ por outros compostos, capazes de fornecer adequadas concentrações de sódio à ave e melhorar a qualidade da casca dos ovos. Uma alternativa tem sido a utilização de bicarbonato de sódio, que apresenta em sua constituição, segundo Hooge (1999), 27,1\% de sódio e $71,9 \%$ de bicarbonato.
Austic \& Kerhavarz (1988), aumentando a proporção de sódio em relação ao cloreto, pela adição de bicarbonato de sódio as dietas, observaram melhora significativa na espessura e resistência da casca dos ovos. Todavia, Schafhauser et al. (2001), estudando o efeito da utilização de sal comum e bicarbonato de sódio para poedeiras Lohmann, com 75 semanas de idade, não verificaram diferença entre a utilização do sal comum, bicarbonato de sódio e sal comum + bicarbonato, sobre o desempenho produtivo e qualidade da casca dos ovos.

A deficiência de sódio nas rações para poedeiras tem provocado grande redução no consumo, produção de ovos, peso dos ovos e peso corporal das aves (Kuchinski et al., 1997). Por outro lado, altos níveis de sódio ocasionam aumentos significativos no consumo de água e, conseqüentemente, elevação da umidade das excretas e excreção urinária de sódio, com significativa redução nas taxas de filtração glomerular (Wideman \& Buss, 1985).

Para poedeiras no primeiro ciclo de produção são várias as recomendações das exigências nutricionais dos eletrólitos, sendo que para Reeve (1990), um bom desempenho das aves no primeiro ciclo de produção pode ser alcançado utilizando-se dietas contendo níveis de sódio entre $0,14-0,28 \%$, entretanto para o NRC (1994) os níveis de $0,15 \%$ de sódio e potássio, e $0,13 \%$ de cloro para poedeiras com ingestão diária de $100 \mathrm{~g}$ de ração e com $2.900 \mathrm{kcal}$ de EM $/ \mathrm{kg}$, seriam os adequados.

Bertechini et al. (1996), estudando poedeiras criadas em clima tropical, durante o pico e o final de postura, concluíram que as exigências de sódio para ótima produção de ovos são 0,150 e $0,180 \%$ e para melhor conversão alimentar são 0,192 e 0,198\%, respectivamente, em cada período estudado.

Segundo Rostagno et al. (2000), as exigências nutricionais de sódio, cloro e potássio para poedeiras leves, com ingestão de $100 \mathrm{~g} /$ ave/dia, são de 0,225 ; 0,200 e $0,580 \%$, respectivamente.

Faria et al. (2000), utilizando níveis de 0,16;0,20 e $0,24 \%$ de sódio e 0,$35 ; 0,45$ e $0,55 \%$ de fósforo para poedeiras leves, às 60 semanas de idade, não observaram efeito dos níveis de sódio sobre o desempenho e qualidade da casca dos ovos. Esses autores concluíram que níveis de sódio mais elevados que $0,16 \%$ não são vantajosos para melhorar o desempenho e a qualidade dos ovos, considerando o padrão de consumo de ração e os níveis de $\mathrm{P}$ adotados por eles.

Junqueira et al. (2000), estudando o efeito da 
utilização de diferentes fontes de sódio $(\mathrm{Na})$, cloro (Cl) e potássio $(\mathrm{K})$, bem como os níveis desses eletrólitos na dieta e a relação $(\mathrm{Na}+\mathrm{K}) / \mathrm{Cl}$, para poedeiras Hy-Line branca, com 54 semanas de idade, observaram melhor peso dos ovos com a relação de 3,46 e 4,46 , utilizando os níveis de $0,16 \% \mathrm{Na}, 0,20 \% \mathrm{Cl}$ e $0,73 \% \mathrm{~K}$ e $0,17 \% \mathrm{Na}, 0,26 \% \mathrm{Cl}$ e $0,73 \% \mathrm{~K}$, respectivamente.

Rodrigues et al. (2002) avaliando o efeito dos níveis de sódio $(0,15 ; 0,25$ e $0,35 \%)$ para poedeiras Hy-Line W-36 pós-muda forçada com 60 semanas de idade, concluíram que os níveis de $0,15 \%$ de sódio no período de repouso e $0,35 \%$ no período de postura são suficientes para atender as exigências nutricionais destas aves, proporcionando bom desempenho produtivo e boa espessura da casca.

Devido à importância nutricional do sódio e suas diversas relações com outros eletrólitos, na determinação de um correto balanço eletrolítico da dieta, sobre a produção e a qualidade dos ovos das galinhas em postura, realizaram-se estes trabalhos. Objetivou-se avaliar os níveis de sódio e balanços eletrolíticos da dieta sobre o desempenho produtivo e a qualidade externa dos ovos de poedeiras comerciais no primeiro e segundo ciclos de produção.

\section{Material e Métodos}

Dois experimentos foram realizados no setor de avicultura da Fazenda Experimental de Iguatemi, na Universidade Estadual de Maringá.

Os experimentos tiveram duração de 112 dias, divididos em quatro ciclos de 28 dias cada. Foram utilizadas 160 poedeiras Lohmann com 25 semanas de idade no experimento I e 160 poedeiras Babcok pós-muda forçada, com 80 semanas de idade no segundo experimento.

As temperaturas médias máximas e mínimas coletadas durante os períodos experimentais foram de 28,3 e $19,4^{\circ} \mathrm{C}$ e 28,1 e $19,6^{\circ} \mathrm{C}$, para os experimentos I e II, respectivamente.

As aves foram distribuídas em um delineamento inteiramente casualizado com cinco tratamentos e quatro repetições de oito aves em cada unidade experimental.

As rações experimentais foram formuladas à base de milho e farelo de soja, sendo todas isoprotéicas, isocalóricas, isoaminoacídicas para Met + Cis e Lisina, isocálcicas e isofosfóricas (Tabela 1), segundo as exigências das linhagens e utilizando-se os valores de composição química e energética dos alimentos segundo Rostagno et al. (1985).

O balanço eletrolítico das dietas (BED) experimentais foi calculado segundo Mongin (1980), por intermédio da fórmula: $\mathrm{N}^{\circ}$ de Mongin $(\mathrm{NM})=\mathrm{mEqNa}^{+}$ $+\mathrm{mEqK}^{+}-\mathrm{mEqCl}^{-} \quad(\mathrm{mEq} / \mathrm{kg})$.

Para o cálculo do NM, a partir de valores percentuais dos eletrólitos, foi empregada a seguinte fórmula: $\mathrm{NM}=\% \mathrm{Na}^{+} \times 10.000 / 22,990 *+\% \mathrm{~K}^{+} \mathrm{x}$ $10.000 / 39,102 *-\% \mathrm{Cl}^{-} \times 10.000 / 35,453 *$ (* Equivalente grama do $\mathrm{Na}, \mathrm{K} \mathrm{e} \mathrm{Cl}$, respectivamente).

Os tratamentos foram constituídos de uma dieta basal deficiente em sódio contendo 0,12 e $0,13 \%$ para os experimentos I e II, respectivamente, suplementadas com cinco níveis de sódio provenientes do bicarbonato de sódio e sal comum, sendo que os níveis empregados foram de 0,$12 ; 0,15 ; 0,18 ; 0,21$, $0,24 \%$ e 0,$13 ; 0,15 ; 0,17 ; 0,19,0,21 \%$ e os balanços eletrolíticos calculados, de 205, 218, 231, 243, 246 $\mathrm{mEq} / \mathrm{kg}$ e 174, 183, 192, 200, $209 \mathrm{mEq} / \mathrm{kg}$, respectivamente, para os experimentos I e II.

Os parâmetros avaliados foram semelhantes para os dois experimentos. O consumo de ração (CR) (g/ave/dia) foi controlado a cada ciclo de 28 dias. A ração correspondente a cada unidade experimental foi pesada e armazenada em baldes plásticos identificados. No $28^{\circ}$ dia de cada ciclo, a sobra de ração do comedouro de cada unidade foi devolvida ao balde correspondente, pesada e, por diferença, determinado o consumo de ração.

Os ovos foram coletados diariamente e anotados por gaiola para determinação da produção total de ovos e porcentagem de postura. Nos quatro últimos dias de cada ciclo todos os ovos foram pesados individualmente em balança de precisão digital $(0,01 \mathrm{~g})$ e posteriormente analisada a qualidade da casca dos ovos pelo método não destrutivo (gravidade específica) e destrutivo (\% casca).

O teste para gravidade específica foi realizado logo após a coleta dos ovos, por imersão dos mesmos em diferentes soluções salinas, com densidades de 1,070; 1,074; 1,078; 1,082 e 1,086 g/mL. As soluções salinas foram ajustadas com a utilização de um densímetro de petróleo e derivados líquidos, sendo calibradas a cada 60 ovos submersos, conforme a recomendação de Moreng \& Avens (1990).

Para determinação da percentagem de casca dos ovos, foram coletados uma amostra de 3 ovos/ parcela/dia, os quais foram identificados, pesados e 
Tabela 1 - Composição percentual e calculada das dietas experimentais

Table 1 - Percentual and calculated composition of the experimental diets

\begin{tabular}{|c|c|c|c|c|c|c|c|c|c|c|}
\hline \multirow[t]{3}{*}{$\begin{array}{l}\text { Ingredientes } \\
\text { Ingredients }\end{array}$} & \multicolumn{10}{|c|}{$\begin{array}{l}\text { Níveis de sódio (\%) } \\
\text { Sodium levels (\%) }\end{array}$} \\
\hline & \multicolumn{5}{|c|}{$\begin{array}{l}\text { Experimento } 1 \\
\text { Experiment } 1\end{array}$} & \multicolumn{5}{|c|}{$\begin{array}{l}\text { Experimento } 2 \\
\text { Experiment } 2\end{array}$} \\
\hline & 0,12 & 0,15 & 0,18 & 0,21 & 0,24 & 0,13 & 0,15 & 0,17 & 0,19 & 0,21 \\
\hline $\begin{array}{l}\text { Milho, grão } \\
\text { Corn, grain }\end{array}$ & 61,70 & 61,53 & 61,41 & 61,20 & 61,00 & 64,22 & 64,07 & 63,93 & 63,79 & 63,63 \\
\hline $\begin{array}{l}\text { Farelo de soja }(45 \%) \\
\text { Soybean meal }\end{array}$ & 27,98 & 27,99 & 28,00 & 28,02 & 28,03 & 24,10 & 24,11 & 24,13 & 24,15 & 24,18 \\
\hline $\begin{array}{l}\text { Fosfato bicálcico } \\
\text { Dicalcium phosphate }\end{array}$ & 1,86 & 1,86 & 1,86 & 1,86 & 1,86 & 1,51 & 1,51 & 1,51 & 1,51 & 1,51 \\
\hline $\begin{array}{l}\text { Calcário calcítico } \\
\text { Limestone }\end{array}$ & 7,77 & 7,77 & 7,77 & 7,77 & 7,77 & 9,60 & 9,60 & 9,60 & 9,60 & 9,60 \\
\hline $\begin{array}{l}\text { Óleo vegetal } \\
\text { Vegetable oil }\end{array}$ & 0,05 & 0,10 & 0,10 & 0,17 & 0,25 & 0,02 & 0,08 & 0,12 & 0,17 & 0,23 \\
\hline $\begin{array}{l}\text { DL-Metionina } 99 \% \\
\text { DL-Methionine } 99 \%\end{array}$ & 0,13 & 0,13 & 0,13 & 0,13 & 0,13 & 0,09 & 0,09 & 0,09 & 0,09 & 0,09 \\
\hline $\begin{array}{l}\text { Sup. vit.-min. }{ }^{1} \\
\text { Vit-min supplement }{ }^{1}\end{array}$ & 0,20 & 0,20 & 0,20 & 0,20 & 0,20 & 0,20 & 0,20 & 0,20 & 0,20 & 0,20 \\
\hline $\begin{array}{l}\text { Antioxidante } \\
\text { Antioxidant }\end{array}$ & 0,01 & 0,01 & 0,01 & 0,01 & 0,01 & 0,01 & 0,01 & 0,01 & 0,01 & 0,01 \\
\hline $\begin{array}{l}\text { Sal comum } \\
\text { Common salt }\end{array}$ & 0,15 & 0,15 & 0,15 & 0,15 & 0,15 & 0,21 & 0,21 & 0,21 & 0,21 & 0,21 \\
\hline $\begin{array}{l}\mathrm{NaHCO}_{3} \\
\text { Valores calculados } \\
\text { Calculated values }\end{array}$ & 0,16 & 0,27 & 0,38 & 0,49 & 0,60 & 0,04 & 0,12 & 0,19 & 0,26 & 0,34 \\
\hline $\begin{array}{l}\text { Proteína bruta (\%) } \\
\text { Crude protein }\end{array}$ & 17,35 & 17,35 & 17,35 & 17,35 & 17,35 & 16,50 & 16,50 & 16,50 & 16,50 & 16,50 \\
\hline $\begin{array}{l}\mathrm{EM}(\mathrm{kcal} / \mathrm{kg}) \\
M E\end{array}$ & 2.750 & 2.750 & 2.750 & 2.750 & 2.750 & 2.750 & 2.750 & 2.750 & 2.750 & 2.750 \\
\hline $\begin{array}{l}\text { Cálcio }(\%) \\
\text { Calcium }\end{array}$ & 3,50 & 3,50 & 3,50 & 3,50 & 3,50 & 4,10 & 4,10 & 4,10 & 4,10 & 4,10 \\
\hline $\begin{array}{l}\text { Fósforo disponível (\%) } \\
\text { Available phosphorus }\end{array}$ & 0,45 & 0,45 & 0,45 & 0,45 & 0,45 & 0,38 & 0,38 & 0,38 & 0,38 & 0,38 \\
\hline $\begin{array}{l}\text { Cloro }(\%) \\
\text { Chloride }\end{array}$ & 0,13 & 0,13 & 0,13 & 0,13 & 0,13 & 0,16 & 0,16 & 0,16 & 0,16 & 0,16 \\
\hline $\begin{array}{l}\text { Potássio (\%) } \\
\text { Potassium }\end{array}$ & 0,74 & 0,74 & 0,74 & 0,74 & 0,74 & 0,64 & 0,64 & 0,64 & 0,64 & 0,64 \\
\hline $\begin{array}{l}\mathrm{BED}(\mathrm{mEq} / \mathrm{kg}) \\
D E B\end{array}$ & 205 & 218 & 231 & 243 & 256 & 174 & 183 & 192 & 200 & 209 \\
\hline
\end{tabular}

quebrados. As cascas foram lavadas e secas à temperatura ambiente por 48 horas. Em seguida foram pesadas e calculada a porcentagem de casca em relação ao peso dos ovos. Posteriormente, mediuse a espessura dessas cascas por meio de um micrômetro manual da marca Mitutoyo de 0-25 mm, com precisão de $0,1 \mathrm{~mm}$.

Ao término do primeiro experimento, foram colocados plásticos sob as gaiolas para coleta das excretas, após 8 horas as mesmas foram recolhidas, identificadas e secas em estufa de $55^{\circ} \mathrm{C}$ durante
72 horas, para posterior determinação da porcentagem de umidade.

Os dados obtidos nos dois experimentos foram submetidos à análise de regressão polinomial, utilizando-se o programa computacional SAEG (1983).

\section{Resultados e Discussão}

Os resultados médios de desempenho das poedeiras no primeiro e segundo ciclos de produção estão apresentados nas Tabelas 2 e 3 , respectivamente. 
Tabela 2 - Desempenho de poedeiras Lohmann no primeiro ciclos de produção, alimentadas com dietas contendo diferentes níveis de sódio e balanços eletrolíticos (BE)

Table 2 - Performance of Lohmann laying hens in the first production cycles, fed diets containing different sodium levels and electrolyte balances (EB)

\begin{tabular}{|c|c|c|c|c|c|c|}
\hline $\begin{array}{l}\text { Níveis de } \mathrm{Na}(\%) \\
\text { Na levels }\end{array}$ & 0,12 & 0,15 & 0,18 & 0,21 & 0,24 & $C V(\%)$ \\
\hline $\begin{array}{l}\text { Produção de ovos (\%) } \\
\text { Egg production }\end{array}$ & 92,21 & 95,64 & 94,53 & 93,56 & 95,20 & 3,23 \\
\hline $\begin{array}{l}\text { Consumo de ração (g/ave/dia) } \\
\text { Feed intake }\end{array}$ & 105,16 & 109,19 & 107,11 & 109,04 & 106,42 & 2,98 \\
\hline $\begin{array}{l}\text { Conversão alimentar }(\mathrm{kg} / \mathrm{kg}) \\
\text { Feed:gain ratio }\end{array}$ & 1,850 & 1,864 & 1,856 & 1,851 & 1,818 & 2,43 \\
\hline $\begin{array}{l}\text { Conversão alimentar }(\mathrm{kg} / \mathrm{dz}) \\
\text { Feed:gain ratio }\end{array}$ & 1,367 & 1,383 & 1,372 & 1,388 & 1,353 & 2,28 \\
\hline $\begin{array}{l}\text { Peso do ovo }(\mathrm{g}) \\
\text { Egg weight }\end{array}$ & 61,58 & 61,80 & 61,65 & 62,53 & 62,11 & 2,73 \\
\hline $\begin{array}{l}\text { Casca }(\%) \\
\text { Eggshell }\end{array}$ & 9,08 & 9,06 & 9,14 & 9,06 & 9,12 & 1,90 \\
\hline $\begin{array}{l}\text { Espessura da casca (mm) } \\
\text { Shell thickness }\end{array}$ & 0,380 & 0,379 & 0,386 & 0,384 & 0,382 & 1,59 \\
\hline $\begin{array}{l}\text { Gravidade específica }(\mathrm{g} / \mathrm{mL}) \\
\text { Egg specific gravity }\end{array}$ & 1,082 & 1,083 & 1,084 & 1,082 & 1,083 & 0,10 \\
\hline $\begin{array}{l}\text { Umidade excretas }(\%) \\
\text { Excreta moisture }\end{array}$ & 80,94 & 79,59 & 81,88 & 80,44 & 83,18 & 2,10 \\
\hline $\begin{array}{l}\text { Balanço eletrolítico }(\mathrm{mEq} / \mathrm{kg}) \\
\text { Electrolyte balance }\end{array}$ & 205 & 218 & 231 & 243 & 256 & \\
\hline
\end{tabular}

$\mathrm{P}>0,05$.

Não houve efeito $(\mathrm{P}>0,05)$ dos níveis de sódio utilizados sobre as variáveis referentes ao desempenho produtivo, à qualidade da casca do ovo e umidade das excretas, concordando com o obtido por Faria et al. (2000), os quais utilizaram níveis de 0,$16 ; 0,20$ e $0,24 \%$ de sódio, associados a níveis de 0,$35 ; 0,45$ e $0,55 \%$ de fósforo, para poedeiras leves, as 60 semanas de idade.

De acordo com os resultados obtidos, verificou-se que os níveis de 0,12 e $0,13 \%$ de sódio foram suficientes para proporcionar bom desempenho produtivo das aves e adequada qualidade da casca dos ovos no primeiro e segundo ciclos de produção, respectivamente, apresentando-se próximos ao sugerido por Scott et al. (1982), os quais recomendam o nível de $0,12 \%$ de sódio para poedeiras em produção.

Esses resultados demonstram que os níveis de sódio utilizados nas rações de poedeiras comerciais podem ser menores do que os recomendados por outros autores (Reeve, 1990; NRC, 1994; Bertechini et al., 1996; Rostagno et al., 2000; Faria et al., 2000; Junqueira etal., 2000; Murakami et al., 2001; Rodrígues et al., 2002), cujas recomendações acerca do nível de sódio ótimo para proporcionar adequado desempenho e qualidade da casca dos ovos de poedeiras comerciais variam de 0,14 a $0,35 \%$, todavia, esses autores não estudaram níveis menores que $0,14 \% \mathrm{Na}$.

Não houve efeito $(\mathrm{P}>0,05)$ dos níveis de sódio sobre a umidade das excretas das poedeiras no primeiro ciclo de postura, concordando com os resultados obtidos por Murakami et al. (2001). Esses autores ao determinarem as exigências nutricionais de sódio $(0,15 ; 0,18 ; 0,21$ e $0,24 \%)$ e cloro $(0,14 ; 0,17$; 0,20 e $0,23 \%$ ) para poedeiras Lohmann com 31 semanas de idade, não constataram efeito significativo dos níveis de sódio sobre a umidade das excretas, todavia concluíram que o nível de $0,24 \%$ de sódio, para baixos $(0,14 \%)$ ou altos $(0,23 \%)$ níveis de cloro, proporcionam menor umidade das excretas e melhor qualidade da casca do ovo. Apesar da recomendação desses autores, os resultados observados no presente trabalho evidenciaram que o nível de $0,12 \%$ de sódio foi suficiente em proporcionar adequada umidade das excretas, sem influenciar negativamente os demais parâmetros.

As diferentes recomendações de sódio para poedeiras, verificadas na literatura, podem ser atribuídas à utilização de aves de diferentes linhagens ou idades, condições ambientais variadas e efeito nutricional dos demais componentes da dieta, uma vez que a associação desses fatores pode proporcionar diferentes respostas aos parâmetros avaliados, 
Tabela 3 - Desempenho de poedeiras Babcok pós muda forçada, alimentadas com dietas contendo diferentes níveis de sódio e balanços eletrolíticos (BE)

Table 3 - Performance of Babckok forced molting hens fed diets containing different sodium levels and different electrolyte balance

\begin{tabular}{|c|c|c|c|c|c|c|}
\hline $\begin{array}{l}\text { Níveis de } \mathrm{Na}(\%) \\
\text { Na levels }\end{array}$ & 0,12 & 0,15 & 0,18 & 0,21 & 0,24 & $\mathrm{CV}(\%)$ \\
\hline $\begin{array}{l}\text { Produção de ovos (\%) } \\
\text { Egg production }\end{array}$ & 73,11 & 69,36 & 78,57 & 70,62 & 69,98 & 7,30 \\
\hline $\begin{array}{l}\text { Consumo de ração (g/ave/dia) } \\
\text { Feed intake }\end{array}$ & 105,76 & 104,59 & 108,77 & 106,21 & 106,60 & 2,02 \\
\hline $\begin{array}{l}\text { Conversão alimentar }(\mathrm{kg} / \mathrm{kg}) \\
\text { Feed:gain ratio }\end{array}$ & 2,212 & 2,214 & 2,018 & 2,219 & 2,167 & 6,43 \\
\hline $\begin{array}{l}\text { Conversão alimentar }(\mathrm{kg} / \mathrm{dz}) \\
\text { Feed:gain ratio }\end{array}$ & 1,769 & 1,804 & 1,657 & 1,809 & 1,768 & 7,12 \\
\hline $\begin{array}{l}\text { Peso do ovo }(\mathrm{g}) \\
\text { Egg weight }\end{array}$ & 66,76 & 67,93 & 68,90 & 68,04 & 68,00 & 2,68 \\
\hline $\begin{array}{l}\text { Casca }(\%) \\
\text { Eggshell }\end{array}$ & 8,64 & 8,45 & 8,58 & 8,58 & 8,52 & 3,18 \\
\hline $\begin{array}{l}\text { Espessura da casca }(\mathrm{mm}) \\
\text { Shell thickness }\end{array}$ & 0,382 & 0,379 & 0,383 & 0,382 & 0,380 & 2,44 \\
\hline $\begin{array}{l}\text { Gravidade específica }(\mathrm{g} / \mathrm{mL}) \\
\text { Egg specific gravity }\end{array}$ & 1,078 & 1,078 & 1,078 & 1,078 & 1,078 & 0,19 \\
\hline $\begin{array}{l}\text { Balanço eletrolítico }(\mathrm{mEq} / \mathrm{kg}) \\
\text { Electrolyte balance }\end{array}$ & 174 & 183 & 192 & 200 & 209 & \\
\hline
\end{tabular}

$\mathrm{P}>0,05$.

durante a determinação do correto nível de sódio para essas aves, levando o nutricionista à tomada de decisão adequada à sua condição.

Na determinação do melhor balanço eletrolítico da dieta, não se observou efeito $(\mathrm{P}>0,05)$ dos mesmos sobre os parâmetros avaliados nos experimentos realizados. Este resultado está de acordo com Junqueira et al. (2000), os quais realizaram dois trabalhos utilizando poedeiras Hy-Line brancas, com 54 semanas de idade, alimentadas com dietas com balanços eletrolíticos variando de 187 a $491 \mathrm{mEq} / \mathrm{kg}$ e não constataram influência dos mesmos sobre o desempenho produtivo e qualidade da casca dos ovos.

Os balanços eletrolíticos de $205 \mathrm{mEq} / \mathrm{kg}$ para poedeiras no primeiro ciclo e $174 \mathrm{mEq} / \mathrm{kg}$ para poedeiras pós muda forçada demonstraram-se eficazes em proporcionar ótima resposta produtiva e boa qualidade da casca dos ovos.

A recomendação do balanço eletrolítico de $205 \mathrm{mEq} / \mathrm{kg}$ para poedeiras no primeiro ciclo de produção concorda com o sugerido por Reeve (1990), de que um balanço eletrolítico acima de $190 \mathrm{mEq} / \mathrm{kg}$ seria suficiente para proporcionar adequada qualidade da casca dos ovos.

Para poedeiras pós muda forçada, o balanço eletrolítico ideal foi de $174 \mathrm{mEq} / \mathrm{kg}$, demonstrando que as aves nesse ciclo de produção podem apresentar exigências nutricionais especificas, as quais dife- rem das aves no primeiro ciclo, como já afirmaram Christmas \& Harms (1983). Esse resultado está de acordo com o recomendado por Judice et al. (2002), que estudaram a influência do balanço cátion-aniônico das rações de poedeiras no segundo ciclo de produção e concluíram que o balanço de $174,5 \mathrm{mEq} / \mathrm{kg}$ (rações catiônicas com 3,8\% de cálcio) proporciona melhor produção de ovos e qualidade da casca, com redução das perdas dos ovos.

O aumento na proporção de sódio em relação ao cloreto, pela adição de níveis crescentes de bicarbonato de sódio as dietas, não apresentou efeito $(\mathrm{P}>0,05)$ sobre a produção e qualidade da casca dos ovos, discordando do observado por Austic \& Kerhavarz. (1988), os quais verificaram melhora significativa na espessura e resistência da casca dos ovos, em função do aumento dessa proporção.

\section{Conclusões}

Nas condições em que os experimentos foram realizados, pode-se concluir que os níveis de $0,12 \%$ de sódio, em dietas com $205 \mathrm{mEq} / \mathrm{kg}$, para poedeiras no primeiro ciclo de produção, e de $0,13 \%$ de sódio, em dietas com $174 \mathrm{mEq} / \mathrm{kg}$, para poedeiras no segundo ciclo de produção, foram suficientes para proporcionarem bom desempenho produtivo e boa qualidade externa dos ovos. 


\section{Literatura Citada}

AUSTIC, R.E.; KESHAVARZ, K. Interaction of dietary calcium and chloride and the influence of monovalent minerals on eggshell quality. Poultry Science, v.67, p.750-759, 1988.

BERTECHINI, A.G.; LIRA, V.M.C.; FASSANI, E.J. Effects of dietary sodium level on performance and egg quality of laying hens in the tropical climate. Official Journal of the Poultry Science Association, v.75, n.1, p.167, 1996. (Supplement 1)

CHRISTMAS, R.B.; HARMS, R.H. The performance of four strains of laying hens subjected to various postrest combinations of calcium and phosphorus after forced rest in winter or summer. Poultry Science, v.62, n.9, p.1816-1822, 1983.

EUCLYDES, R.S. Manual de utilização do programa SAEG (Sistema para Análise Estatística e Genética). Viçosa UFV, 59p, 1983.

FARIA, D.E.; JUNQUEIRA, O.M.; SAKOMURA, N.K. et al. Efeito de diferentes níveis de sódio e fósforo sobre o desempenho e a qualidade da casca dos ovos de poedeiras comerciais. Revista Brasileira de Zootecnia, v.29, n.2, p.458466, 2000

HOOGE, D.M. A importância dos eletrólitos. Avicultura Industrial, n.1068, 1999, p.20-26.

JUDICE, J.P.M.; BERTECHINI, A.G.; MUNIZ, J.A. et al. Balanço cátio-aniônico das rações e manejo alimentar para poedeiras de segundo ciclo. Lavras. Ciência Agrotécnica, v.26, n.3, p.598-609, 2002.

JUNQUEIRA, O.M.; CAMARGO-FILHO, B.; ARAUJO, L.F. et al. Efeitos das fontes e níveis de sódio, cloro e potássio e da relação $(\mathrm{Na}+\mathrm{K}) / \mathrm{Cl}$, sobre o desempenho e características do plasma sangüíneo de poedeiras comerciais. Revista Brasileira de Zootecnia, v.29, n.4, p.1110-1116, 2000.

KUCHINSKI, K.K.; HARMS, R.H.; RUSSEL, G. Re-evaluation of the sodium of the commercial laying hen. In: ANNUAL MEETING POULTRY SCIENCE, 86, 1997, LousvilleKentucky. Proceedings... Lousville: Supplement 1, v.59, 1997, p.236.

LEESON, S. Feeding programs for laying hens. ASA: American Soybean Association, 2001, n.70, p.9-10 (Technical bulletin Vol. PO49-2001).

MILES, R.D.; ROSSI, A. Cation-anion balance in laying hens. In: FLORIDA NUTRITIONAL CONFERENCE, 1984, Clearwater Beach. Proceedings... Clearwater Beach: University of Florida, 1984. p.15-22.

MONGIN, P. Electrolytes in nutrition: a review of basic principles and practical application in poultry and swine. In: ANNUAL MINESOTTA CONFERENCE, 3., 1980, Illinois. Proceedings... Illinois: IMC, 1980. p.1-15.

MORENG, R.E.; AVENS, J.S.Ciência e produção de aves. São Paulo: Roca, 1990. 380p.

MURAKAMI, A.E.; SAKAMOTO, M.I.; MARTINS, E.N. et al. Requirements of sodium and chloride for leghorn layers hens. In: EUROPE POULTRY NUTRITION, 13., 2001, Blankenberge - Belgium. Prodeedings... Blankenberge: WPSA, 2001.p.194-195.
NATIONAL RESEARCH COUNCIL - NRC. Nutrient requirements of poultry. 9.ed. Washington: National Academic Press, 1994. 155p.

OLIVEIRA, B.L. Custo dos pontos críticos da recria e produção de poedeiras - Manejo. In: CONFERÊNCIA APINCO DE CIÊNCIA E TECNOLOGIA AVÍCOLA, 1992, Santos, Anais... Santos: FACTA, 1992. p.137-144.

REEVE, A. The role of sodium bicarbonate in improving egg shell quality. Manage Ment Guide: Institut de Selection Animale, 1990. p.1-7 (Technical note, 19).

RODRIGUES, E.A.; JUNQUEIRA, O.M.; VALÉRIO, M. et al. 2002. Níveis de sódio em rações de poedeiras comerciais no segundo ciclo de postura. In: REUNIÃO ANUAL DA SOCIEDADE BRASILEIRA DE ZOOTECNIA, 39., 2002, Recife. Anais... Recife: Sociedade Brasileira de Zootecnia/ technoMIDIA, [2002] 05par. CD-ROM. Nutrição de Não Ruminates. NNR-385.

RostagnO, H.S.; SILVA, D.J.; COSTA, P.M.A. Composição de alimentos e exigências nutricionais de aves e suínos (tabelas brasileiras). Viçosa, MG: Universidade Federal de Viçosa, 1985. 50p.

ROSTAGNO, H.S.; ALBINO, L.F.T.; DONZELE, J.L. et al. Tabelas brasileiras para aves e suínos; composição de alimentos e exigências nutricionais.Viçosa, MG: Universidade Federal de Viçosa, 2000. 141p.

SCOTT, M.L.; NESHEIN, M.C.; YOUNG, R.J.; Nutrition of the chichen. 3.ed. Ithaca: Scott Scott and Associates, 1982. 562 p.

SHAFHAUSER, D.; SAKAMOTO, M.I.; GALLI, J.R. et al. Efeito da utilização de sal comum e bicarbonato de sódio para poedeiras comerciais. In: ENCONTRO ANUAL DE INICIAÇÃO CIENTÍfICA, 10., 2001, Ponta Grossa. Anais... Ponta Grossa: UEPG, 2001. p.360-361.

VICENZI, E. Fadiga de gaiola e qualidade da casca do ovo Aspectos nutricionais. In: SIMPÓSIO TÉCNICO DE PRODUÇÃO DE OVOS, 6., 1996, São Paulo. Anais... São Paulo: APA, 1996. p.77-91.

WIDEMAN, R.F.; BUSS, E.G. Arterial blood gas, pH, and bicarbonate values in laying hens for thin eggshell production. Poultry Science, v.64, p.1015-1019, 1985.

YOSELEWITZ, A.; BALNAVE, D.. The influence of saline drink ink water on the activity in the shell gland of laying hens. Australian Journal Agricultural Researches, v.40, p.1111-1115, 1989.
Recebido em: 09/10/02 Aceito em: 10/04/03 